\title{
Interesting Conjugate Points in Formation Constrained Optimal Multi-Agent Coordination
}

\author{
Jianghai Hu, Maria Prandini and Claire Tomlin
}

\begin{abstract}
In this paper, an optimal coordinated motion planning problem is formulated where multiple agents have to reach given destination positions starting from given initial positions, subject to constraints on the admissible formation patterns. Solutions to the problem are reinterpreted as distanceminimizing geodesics on a certain manifold with boundary. A geodesic on this manifold that possesses conjugate points will not be distance-minimizing beyond its first conjugate point. We study a particular instance of the problem, where a number of initially aligned agents tries to switch positions by rotating around their common centroid. We characterize analytically the complete set of conjugate points of a geodesic that naturally arises as a candidate solution. This allows us to prove that the geodesic does not correspond to an optimal coordinated motion when the angle of rotation exceeds a threshold that decreases to zero as the number of agents increases. Moreover, infinitesimal perturbations that improve the performance of the geodesic after it passes the conjugate points are characterized by a family of orthogonal polynomials.
\end{abstract}

\section{INTRODUCTION}

In this paper, we formulate an optimal coordinate motion planning problem for multiple agents under formation constraints. Multi-agent coordinated motion planning problems arise in various contexts, such as Air Traffic Management (ATM [1]), robotics ([2]), and Unmanned Aerial Vehicles (UAVs [3], [4], [5]). In most cases, certain separation constraints between the agents have to be guaranteed due to physical, safety, or efficiency reasons. For example, in ATM systems, aircraft flying at the same altitude are required to maintain a minimal horizontal separation of 5 nautical miles (nmi) in en-route airspace and $3 \mathrm{nmi}$ close to airports. When multiple mobile robots are performing a coordinated task such as lifting a common object, specific formations have to be kept by the robots throughout the operation. For UAVs, flying in formation may reduce the fuel expenditure and the communication power needed for information exchange.

In our formulation, we consider all the coordinated motions that lead a group of agents from some initial positions to some destination positions within a given time horizon, while satisfying the additional constraint that the

Research supported by ONR under the MURI project "Computational Methods for Collaborative Motion," and by the European Commission under the project HYBRIDGE IST-2001-32460.

Jianghai $\mathrm{Hu}$ is with the School of Electrical and Computer Engineering, Purdue University, West Lafayette, IN 47906, USA. E-mail: jianghai@purdue.edu.

Maria Prandini is with Dipartimento di Elettronica e Informazione, Politecnico di Milano, Piazza Leonardo da Vinci 32, 20133 Milano, Italy. E-mail: prandini@elet.polimi.it.

Claire Tomlin is with the Department of Aeronautics and Astronautics, Stanford University, Stanford, CA 94305, USA. E-mail: tomlinestanford.edu. formation patterns of the agents during the time interval of interest belong to a prescribed set. Among the coordinated motions in this restricted set, we try to find the ones that minimize a weighted sum of the energy functions of the individual agents' motions, with the weights representing agent priorities. In this formulation, we use simple kinetic models for the agent dynamics, and consider only holonomic constraints, as opposed to contributions dealing with nonholonomic constraints (e.g. [6], [7]). See, e.g., [8], [9], [10], for relevant works on the problems of stable and optimal coordinated control of vehicle formations.

A geometric interpretation of the considered optimal coordinated motion planning problem is given in this paper. According to this interpretation, a solution to the problem is a shortest curve with constant speed between two fixed points in a certain manifold with boundaries: the fixed points represent the starting and destination positions of the agents, whereas the boundaries are determined by the feasible formation patterns. Note that such a globally distanceminimizing curve is also a locally distance-minimizing one, i.e., a curve whose sufficiently short segments are distanceminimizing between their respective end points. Locally distance-minimizing curves parameterized with constant speed are called geodesics. Thus a solution to the problem necessarily corresponds to a geodesic of the manifold.

Conversely, however, a geodesic of the manifold may fail to be globally distance-minimizing for various reasons, so that the corresponding constant speed coordinated motion fails to be a solution to the multi-agent optimal coordination problem. The aim of this paper is to study through a concrete example the loss of optimality due to the occurrence of conjugate points, which so far has been largely ignored in the literature on multi-agent coordination.

Traveling along a geodesic from a fixed starting point, a conjugate point is one at which there exists a non-trivial Jacobi field along the geodesic vanishing at both the starting point and that particular point [11], or less rigorously, where there exists infinitesimally more than one geodesic connecting the starting point to that point. For a simple example, consider the sphere. Geodesics on the sphere are great circles; and conjugate points along a great circle occur at the anti-podal point of its starting point. It is a well known fact in Riemannian geometry that a geodesic will not be distance minimizing once it passes its first conjugate point [11], as one can then infinitesimally perturb it to obtain a shorter curve with the same end points.

It is in general difficult, if not impossible, to characterize the conjugate points of a geodesic analytically. In this 


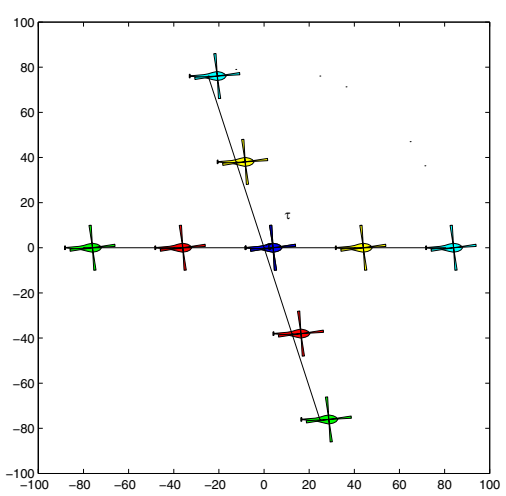

Fig. 1. A five-agent example

paper, we shall focus on a special instance of the formation constrained optimal coordination problem, namely, a group of initially aligned agents switching positions by rotating around their common centroid. We shall show that the conjugate points of a geodesic that arises naturally when solving this particular problem admit nice analytic formulae. We shall also determine the infinitesimal perturbations that can shorten the geodesic with various efficiencies once it passes its conjugate points, and characterize them using a certain family of orthogonal polynomials. Our results can be given the geometric interpretation of how long one can travel along the outer edge of a (high-dimensional) "donut" before the resulting curve is no longer distanceminimizing, or the mechanical interpretation of how a multi-segment snake-like robot should turn on the ground optimally starting from the configuration in which all its segments are aligned in a straight line. See [12] for more details. The result for the three agent case $(k=3$ in the notation of Section II) was first proved in [13].

As a preview of the results and a specific example, see Fig. 1, where five helicopters initially flying in a straight line try to reach new positions by rotating counterclockwise around their centroid, i.e., the middle helicopter, at the same angular velocity during the time horizon $[0,1]$ (thus the five helicopters form a straight line at all times). The results in this paper will show that this joint maneuver is optimal in the sense of minimizing a cost function defined as the sum of energy of individual helicopter's maneuvers only if the angle $\tau$ of rotation is small, and that it is not optimal if $\tau>\frac{\pi}{3}$. Indeed, if for example $\tau=\pi$, then one can find better maneuvers than the one in Fig. 1. In Fig. 2 we plot columnwise three such maneuvers, where in each column the five figures from top to bottom represent the snapshots of the maneuver at time $t=0, \frac{1}{4}, \frac{1}{2}, \frac{3}{4}, 1$, respectively. In terms of performance, we shall show that maneuver (c) is the best of the three; maneuver (a) is the worst of the three, but still better than the original joint maneuver in Fig. 1.

The paper is organized as follows. In Section II the formation patterns of a group of agents are defined and the problem of formation constrained optimal multi-agent coordination is formulated. In Section III the conjugate points

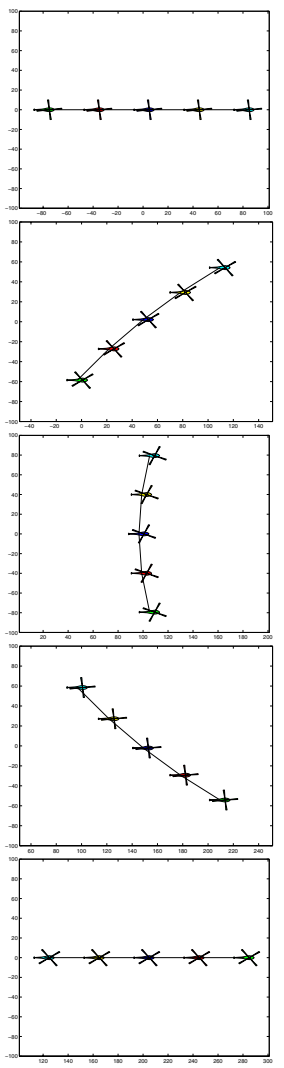

(a)

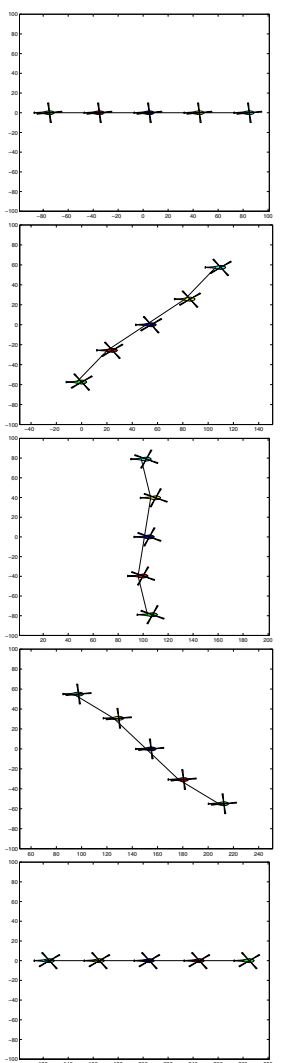

(b)

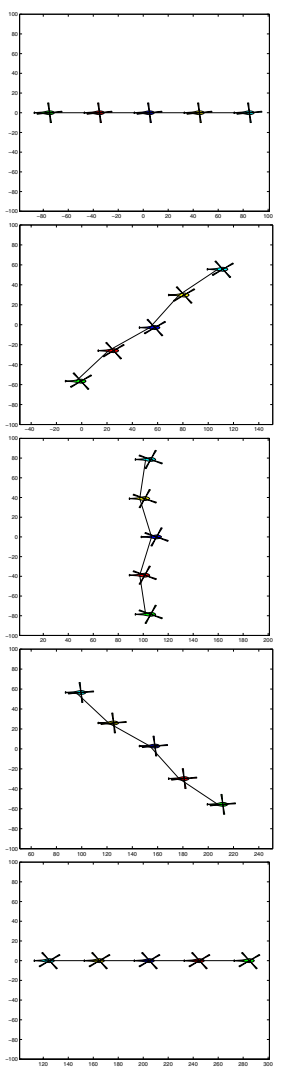

(c)
Fig. 2. Three joint maneuvers with increasingly lower cost than the one shown in Fig. 1 when $\tau=\pi$. Left column: maneuver (a); middle column: maneuver (b); right column: maneuver (c). From top to bottom: snapshots at time $t=0, \frac{1}{4}, \frac{1}{2}, \frac{3}{4}, 1$, respectively.

of the geodesic arising in a particular instance of the multiagent coordination problem are characterized analytically. Infinitesimal perturbations that improve the performance of the geodesic beyond its conjugate points are determined. Finally, some conclusions are drawn in Section IV.

\section{Formation CONSTRAined Optimal Multi-Agent CoORdination}

\section{A. Problem Formulation}

Denote by $\left\langle q_{i}\right\rangle_{i=1}^{k}=\left(q_{1}, \ldots, q_{k}\right)$ an (ordered) $k$-tuple of points in the Euclidean space $\mathbb{R}^{n}$ for some integer $k>0$. We say that $\left\langle q_{i}\right\rangle_{i=1}^{k}$ satisfies the $r$-separation condition for some $r>0$ if $d\left(q_{i}, q_{j}\right)=\left\|q_{i}-q_{j}\right\| \geq r$ for all $i \neq j$, i.e., if the minimum pairwise distance among the $k$ points is at least $r$. Given $\left\langle q_{i}\right\rangle_{i=1}^{k}$ satisfying the $r$-separation constraint, the undirected graph $(\mathcal{V}, \mathcal{E})$ with set of vertices $\mathcal{V}=\{1, \ldots, k\}$ and with set of edges $\mathcal{E}=\{(i, j)$ : $\left.\left\|q_{i}-q_{j}\right\|=r\right\}$ is called the formation pattern of the $k$ tuple $\left\langle q_{i}\right\rangle_{i=1}^{k}$. $(\mathcal{V}, \mathcal{E})$ encodes information on which pairs of points are at the minimal allowed distance $r$. Denote by $\mathcal{F}$ the set of all possible formation patterns.

Consider $k$ agents moving in $\mathbb{R}^{n}$. Suppose that they start at time 0 from the initial positions $a_{1}, \ldots, a_{k} \in \mathbb{R}^{n}$ and 
must reach the destination positions $b_{1}, \ldots, b_{k} \in \mathbb{R}^{n}$ at time $t_{f}$. Denote the joint trajectory of the agents by a $k$-tuple of curves $\gamma=\left\langle\gamma_{i}\right\rangle_{i=1}^{k}$, where the trajectory of agent $i$ during the time interval $\left[0, t_{f}\right]$ is modeled as a continuous and piecewise $C^{1}$ curve $\gamma_{i}:\left[0, t_{f}\right] \rightarrow \mathbb{R}^{n}$ such that $\gamma_{i}(0)=a_{i}$ and $\gamma_{i}\left(t_{f}\right)=b_{i} . \gamma$ is said to be collision-free if for all $t \in\left[0, t_{f}\right]$ the $k$-tuple $\left\langle\gamma_{i}(t)\right\rangle_{i=1}^{k}$ satisfies the $r$-separation condition, or equivalently, if the distance between any two agents is at least $r$ at all times during $\left[0, t_{f}\right]$. We assume that both $\left\langle a_{i}\right\rangle_{i=1}^{k}$ and $\left\langle b_{i}\right\rangle_{i=1}^{k}$ satisfy the $r$-separation condition. The formation pattern of a collision-free joint trajectory $\gamma$ at time $t \in\left[0, t_{f}\right]$ is the formation pattern of $\left\langle\gamma_{i}(t)\right\rangle_{i=1}^{k}$.

Define the cost of the joint trajectory $\gamma$ as

$$
J(\gamma)=\sum_{i=1}^{k} \mu_{i} E\left(\gamma_{i}\right)
$$

where $\mu_{1}, \ldots, \mu_{k}$ are positive numbers representing the priorities of the agents, and

$$
E\left(\gamma_{i}\right)=\frac{1}{2} \int_{0}^{t_{f}}\left\|\dot{\gamma}_{i}(t)\right\|^{2} d t
$$

is the standard energy of $\gamma_{i}$ as a curve in $\mathbb{R}^{n}$, for $i=$ $1, \ldots, k$. Denote by $L\left(\gamma_{i}\right)=\int_{0}^{t_{f}}\left\|\dot{\gamma}_{i}(t)\right\| d t$ the arc length of $\gamma_{i}$. Then $E\left(\gamma_{i}\right) \geq \frac{1}{2} L^{2}\left(\gamma_{i}\right) / t_{f}$ (see [14]), where equality holds if and only if $\left\|\dot{\gamma}_{i}(t)\right\|$ is constant for $t \in\left[0, t_{f}\right]$.

The problem of formation constrained optimal multiagent coordination can now be formulated as follows.

Problem 1 Among all the collision-free joint trajectories $\gamma$ that start from $\left\langle a_{i}\right\rangle_{i=1}^{k}$ at time 0 and end at $\left\langle b_{i}\right\rangle_{i=1}^{k}$ at time $t_{f}$, find the ones that minimize the cost $J(\gamma)$ and satisfy the constraint that the formation pattern of $\gamma$ at any time $t \in\left[0, t_{f}\right]$ belongs to some prescribed subset $\tilde{\mathcal{F}}$ of $\mathcal{F} . \tilde{\mathcal{F}}$ is called the set of admissible formations, and is assumed to contain the formation patterns of both $\left\langle a_{i}\right\rangle_{i=1}^{k}$ and $\left\langle b_{i}\right\rangle_{i=1}^{k}$.

Problem 1 is formulated in Euclidean spaces. Extension to general Riemannian manifolds can be found in [15].

\section{B. Geometric Interpretation}

A geometric interpretation of Problem 1 can be given as follows. Each $k$-tuple $\left\langle q_{i}\right\rangle_{i=1}^{k}$ of points in $\mathbb{R}^{n}$ corresponds to a single point $q=\left(q_{1}, \ldots, q_{k}\right)$ in $\mathbb{R}^{n k}=\mathbb{R}^{n} \times \cdots \times$ $\mathbb{R}^{n}$ ( $k$ times). Thus each joint trajectory $\left\langle\gamma_{i}\right\rangle_{i=1}^{k}$ of the $k$ agents corresponds to a curve $\gamma$ in $\mathbb{R}^{n k}$ starting from $a=$ $\left(a_{1}, \ldots, a_{k}\right)$ at time 0 and ending at $b=\left(b_{1}, \ldots, b_{k}\right)$ at time $t_{f}$. The collision-free condition is equivalent to $\gamma$ avoiding

$$
W \triangleq \cup_{i \neq j}\left\{\left(q_{1}, \ldots, q_{k}\right) \in \mathbb{R}^{n k}:\left\|q_{i}-q_{j}\right\|<r\right\},
$$

or equivalently, $\gamma$ lying in $\mathbb{R}^{n k} \backslash W$, a manifold with boundary. To satisfy the admissible formation constraint, $\gamma$ should further lie in a subset $P$ of $\mathbb{R}^{n k} \backslash W$ obtained by piecing together cells of various dimensions, one for each admissible formation pattern in $\tilde{\mathcal{F}}$. By assumption, $P$ contains both $a$ and $b$.
If $\mu_{1}=\cdots=\mu_{k}=1$, the cost $J(\gamma)$ in (1) is the standard energy of $\gamma$ as a curve in $\mathbb{R}^{n k}$. For general $\left\langle\mu_{i}\right\rangle_{i=1}^{k}$, $J(\gamma)$ is the standard energy of $\gamma$ as a curve in $\mathbb{R}^{n k}$ after appropriately scaling the coordinate axes.

From the above discussions, we conclude that the solutions to Problem 1 are energy-minimizing curves in $P$ from $a$ to $b$. It is well known [11] that such curves are necessarily the shortest curves in $P$ parameterized with constant speed. Therefore, Problem 1 is equivalent to finding the geodesics from $a$ to $b$ in $P$ that are also globally distance-minimizing.

\section{Conservation Law for the Solutions}

Proposition 1 ([15]) Suppose that $\gamma=\left\langle\gamma_{i}\right\rangle_{i=1}^{k}$ is a solution to Problem 1. Then the quantities

$$
\sum_{i=1}^{k} \mu_{i} \dot{\gamma}_{i}(t), \quad \sum_{i=1}^{k} \mu_{i}\left(\gamma_{i}(t) \dot{\gamma}_{i}^{T}(t)-\dot{\gamma}_{i}(t) \gamma_{i}^{T}(t)\right)
$$

are constant for all $t \in\left[0, t_{f}\right]$.

If one thinks of each agent $i$ as a point in $\mathbb{R}^{n}$ with mass $\mu_{i}$, then Proposition 1 implies that the linear and (generalized) angular momenta of the $k$-point mass system are conserved along the solutions to Problem 1. As a result, for a solution $\gamma=\left\langle\gamma_{i}\right\rangle_{i=1}^{k}$ to Problem 1, if both $a$ and $b$ are $\mu$-aligned, i.e., if $\sum_{i=1}^{k} \mu_{i} a_{i}=\sum_{i=1}^{k} \mu_{i} b_{i}=0$, then so is any $k$-tuple $\left\langle\gamma_{i}(t)\right\rangle_{i=1}^{k}, t \in\left[0, t_{f}\right]$, namely, $\sum_{i=1}^{k} \mu_{i} \gamma_{i} \equiv 0$. Thus $\gamma$ as a curve in $\mathbb{R}^{n k}$ must lie in a subspace $V$ of $\mathbb{R}^{n k}$ defined as $V=\left\{\left(q_{1}, \ldots, q_{k}\right) \in \mathbb{R}^{n k}: \sum_{i=1}^{k} \mu_{i} q_{i}=0\right\}$. This reduces the dimension of the state space by $n$.

\section{AN INTERESTING EXAMPLE}

Consider Problem 1 on $\mathbb{R}^{2}$ with the $k$ agents having the same priority $\mu_{1}=\cdots=\mu_{k}=1$ and the minimal allowed separation $r=1$. Suppose that the starting positions $\left\langle a_{i}\right\rangle_{i=1}^{k}$ of the agents are given by $\left\langle\left(\frac{2 i-k-1}{2}, 0\right)\right\rangle_{i=1}^{k}$. In other words, at time $t=0$, the $k$ agents are aligned on the $x$-axis with common centroid at the origin and with consecutive agents at the minimal allowed separation. For each $t \geq 0$, denote by $R_{t}: \mathbb{R}^{2} \rightarrow \mathbb{R}^{2}$ the counterclockwise rotation of $\mathbb{R}^{2}$ by an angle $t$ in radians. Suppose that the destination positions are $\left\langle b_{i}\right\rangle_{i=1}^{k}=\left\langle R_{t_{f}}\left(a_{i}\right)\right\rangle_{i=1}^{k}$. Both the initial and destination positions have the same formation pattern $(\mathcal{V}, \mathcal{E})$, where $\mathcal{V}=\{1, \ldots, k\}$ and $\mathcal{E}=\{(i, i+1): i=1, \ldots, k-1\}$. Choose the admissible formation pattern set $\tilde{\mathcal{F}}$ to consist of this formation pattern only. Thus, in considering Problem 1, we require that agents $i$ and $i+1$ are kept at constant distance $r$ throughout $\left[0, t_{f}\right]$ for $i=1, \ldots, k-1$, and all other pairs of agents maintain a distance greater than $r$.

Due to the admissible formation pattern set $\tilde{\mathcal{F}}$, a solution $\gamma=\left\langle\gamma_{i}\right\rangle_{i=1}^{k}$ to Problem 1 as a curve in $\mathbb{R}^{2 k} \backslash W$ lies in a subset $P$ of $\mathbb{R}^{2 k} \backslash W$ given by

$$
\begin{aligned}
P=\{ & \left(q_{1}, \ldots, q_{k}\right) \in \mathbb{R}^{2 k}:\left\|q_{i}-q_{i+1}\right\|=1,1 \leq i \leq k-1, \\
& \left.\left\|q_{i}-q_{j}\right\|>1, \forall j>i+1\right\} .
\end{aligned}
$$

Since $\left\langle a_{i}\right\rangle_{i=1}^{k}$ and $\left\langle b_{i}\right\rangle_{i=1}^{k}$ are $\mu$-aligned, by the discussion at the end of Section II-C, $\gamma$ lies in the subspace 


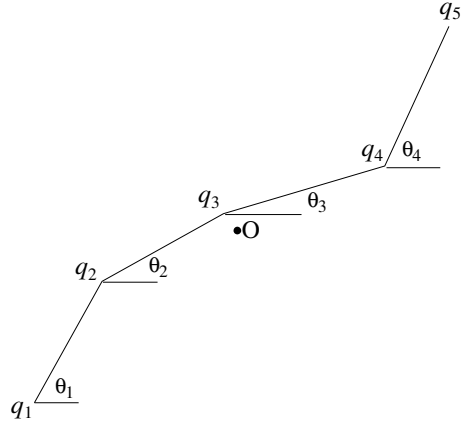

Fig. 3. Coordinates of the manifold $N$ when $k=5$.

$V=\left\{\left(q_{1}, \ldots, q_{k}\right) \in \mathbb{R}^{2 k}: \sum_{i=1}^{k} q_{i}=0\right\}$. Solutions to Problem 1 are then distance-minimizing geodesics in $N=P \cap V$ between $a=\left(a_{1}, \ldots, a_{k}\right)$ and $b=\left(b_{1}, \ldots, b_{k}\right)$.

In this section, we shall consider $\gamma^{*}=\left\langle R_{t}\left(a_{i}\right)\right\rangle_{i=1}^{k}$, $t \in\left[0, t_{f}\right]$, as a natural candidate solution to Problem 1 . According to $\gamma^{*}$, the $k$ agents rotate counterclockwise at constant unit angular velocity around the origin. We shall show that $\gamma^{*}$ is a geodesic in $N$; hence $\gamma^{*}$ is optimal for $t_{f}$ small enough. We shall also show that the first conjugate point along $\gamma^{*}$ occurs at a time $t=\tau_{k}$; hence $\gamma^{*}$ is not optimal if $t_{f}>\tau_{k}$. We shall derive the analytical expression of $\tau_{k}$, and show that $\tau_{k} \sim \frac{1}{k} \rightarrow 0$ as $k \rightarrow \infty$.

\section{A. Geometry of the Manifold $\mathbf{N}$}

We start by studying the geometry of $N$. First of all, $N$ is a $(k-1)$-dimensional smooth submanifold of $\mathbb{R}^{2 k}$, and admits global coordinates $\left(\theta_{1}, \ldots, \theta_{k-1}\right)$, where $\theta_{i}$ is the angle $q_{i+1}-q_{i} \in \mathbb{R}^{2}$ makes with respect to the positive $x$ axis (see Fig. 3). The coordinate map $f:\left(\theta_{1}, \ldots, \theta_{k-1}\right) \mapsto$ $\left(q_{1}, \ldots, q_{k}\right) \in N$ is defined by

$$
q_{i}=q_{1}+\sum_{j=1}^{i-1}\left[\begin{array}{c}
\cos \theta_{j} \\
\sin \theta_{j}
\end{array}\right], \quad i=2, \ldots, k,
$$

where $q_{1}$ is chosen such that $\sum_{i=1}^{k} q_{i}=0$, namely,

$$
q_{1}=-\frac{1}{k} \sum_{j=1}^{k-1}(k-j)\left[\begin{array}{c}
\cos \theta_{j} \\
\sin \theta_{j}
\end{array}\right] .
$$

$\gamma^{*}$ then corresponds to $\theta_{i}(t)=t, t \in\left[0, t_{f}\right], 1 \leq i \leq k-1$.

At any $q \in N, \frac{\partial}{\partial \theta_{1}}, \ldots, \frac{\partial}{\partial \theta_{k-1}}$ form a basis of $T_{q} N$, the tangent space of $N$ at $q$. In this basis, the Riemannian metric $\langle\cdot, \cdot\rangle$ that $N$ inherits from $\mathbb{R}^{2 k}$ as a submanifold is

$$
g_{i j} \triangleq\left\langle\frac{\partial}{\partial \theta_{i}}, \frac{\partial}{\partial \theta_{j}}\right\rangle=\left\langle\frac{\partial f}{\partial \theta_{i}}, \frac{\partial f}{\partial \theta_{j}}\right\rangle_{\mathbb{R}^{2 k}}, 1 \leq i, j \leq k-1 .
$$

Here $f$ is the map defined in (5) and (6), and each $\frac{\partial f}{\partial \theta_{i}}$ is a vector in $\mathbb{R}^{2 k} \cdot\langle\cdot, \cdot\rangle_{\mathbb{R}^{2 k}}$ is the standard inner product on $\mathbb{R}^{2 k}$. Under this metric, the map $f$ becomes an isometry, and the cost of a joint trajectory $\gamma$ of the $k$-agent system given by (1) can be expressed in two equivalent ways:

$J(\gamma)= \begin{cases}\frac{1}{2} \int_{0}^{t_{f}} \sum_{i=1}^{k}\left\|\dot{q}_{i}\right\|^{2} d t & \text { in }\left(q_{1}, \ldots, q_{k}\right) \text { coord. } \\ \frac{1}{2} \int_{0}^{t_{f}} \sum_{i, j=1}^{k-1} g_{i j} \dot{\theta}_{i} \dot{\theta}_{j} d t & \text { in }\left(\theta_{1}, \ldots, \theta_{k}\right) \text { coord. }\end{cases}$
After some careful computation, (7) in our case yields

$$
g_{i j}=\Delta_{i j} \cos \left(\theta_{i}-\theta_{j}\right),
$$

where $\Delta_{i j}$ are constants given by

$$
\Delta_{i j}= \begin{cases}\frac{i(k-j)}{k} & \text { if } i \leq j, \\ \frac{(k-i) j}{k} & \text { if } i>j .\end{cases}
$$

The following lemma can be verified directly.

Lemma 1 Let $\Delta=\left(\Delta_{i j}\right)_{1 \leq i, j \leq k-1} \in \mathbb{R}^{(k-1) \times(k-1)}$ be the symmetric matrix defined in (8). Then

$$
\Delta^{-1}=\left[\begin{array}{ccccc}
2 & -1 & & & \\
-1 & 2 & -1 & & \\
& \ddots & \ddots & \ddots & \\
& & -1 & 2 & -1 \\
& & & -1 & 2
\end{array}\right] .
$$

Denote by $\left(g^{i j}\right)_{1 \leq i, j \leq k-1}$ the inverse matrix of $\left(g_{i j}\right)_{1 \leq i, j \leq k-1}$. The covariant derivative with respect to the Levi-Civita connection on $N$ is given by [11]

$$
\nabla_{\frac{\partial}{\partial \theta_{i}}} \frac{\partial}{\partial \theta_{j}}=\sum_{m=1}^{k-1} \Gamma_{i j}^{m} \frac{\partial}{\partial \theta_{m}},
$$

where $\Gamma_{i j}^{m}$ are the Christoffel symbols defined as

$\Gamma_{i j}^{m}=\frac{1}{2} \sum_{l=1}^{k-1}\left\{\frac{\partial g_{j l}}{\partial \theta_{i}}+\frac{\partial g_{l i}}{\partial \theta_{j}}-\frac{\partial g_{i j}}{\partial \theta_{k}}\right\} g^{l m}, 1 \leq i, j, m \leq k-1$.

A curve $\gamma$ in $N$ is a geodesic if and only if $\nabla_{\dot{\gamma}} \dot{\gamma} \equiv 0$. By definition (10), this equation (known as the geodesic equation) can be written in the $\left(\theta_{1}, \ldots, \theta_{k-1}\right)$ coordinates as a group of second order differential equations:

$$
\ddot{\theta}_{m}=\sum_{i, j=1}^{k-1} \Gamma_{i j}^{m} \dot{\theta}_{i} \dot{\theta}_{j}, \quad m=1, \ldots, k-1 .
$$

In our case, we can compute that, for $1 \leq i, j, m \leq k-1$,

$$
\Gamma_{i j}^{m}= \begin{cases}0 & \text { if } i \neq j, \\ \sum_{l=1}^{k-1} \Delta_{i l} \sin \left(\theta_{l}-\theta_{i}\right) g^{l m} & \text { if } i=j .\end{cases}
$$

Note that along $\gamma^{*}$ we have $\theta_{1}=\cdots=\theta_{k-1}$. Therefore,

Lemma 2 Along $\gamma^{*}$ we have $\Gamma_{i j}^{m}=0$ for all $i, j, m$, hence $\nabla_{\frac{\partial}{\partial \theta_{i}}} \frac{\partial}{\partial \theta_{j}}=0$ for all $i, j$.

Since $\dot{\gamma}^{*}=\frac{\partial}{\partial \theta_{1}}+\cdots+\frac{\partial}{\partial \theta_{k-1}}$, by Lemma 2 and the linearity of covariant derivatives,

$$
\nabla_{\dot{\gamma}^{*}} \dot{\gamma}^{*}=\sum_{i, j=1}^{k-1} \nabla_{\frac{\partial}{\partial \theta_{i}}} \frac{\partial}{\partial \theta_{j}}=0 .
$$

This is exactly the condition for $\gamma^{*}$ to be a geodesic in $N$.

Corollary $1 \gamma^{*}$ is a geodesic in $N$.

Since a geodesic is locally distance-minimizing, $\gamma^{*}$ is a solution to Problem 1 for $t_{f}$ small enough. 


\section{B. Conjugate Points along $\gamma^{*}$}

The curvature tensor of $N$ is defined by [11]

$$
R\left(\frac{\partial}{\partial \theta_{i}}, \frac{\partial}{\partial \theta_{j}}\right) \frac{\partial}{\partial \theta_{l}}=\sum_{m=1}^{k-1} R_{i j l}^{m} \frac{\partial}{\partial \theta_{m}},
$$

where $R_{i j l}^{m}$ are defined from the Christoffel symbols as

$$
R_{i j l}^{m}=\sum_{\beta=1}^{k-1} \Gamma_{i l}^{\beta} \Gamma_{j \beta}^{m}-\sum_{\beta=1}^{k-1} \Gamma_{j l}^{\beta} \Gamma_{i \beta}^{m}+\frac{\partial \Gamma_{i l}^{m}}{\partial \theta_{j}}-\frac{\partial \Gamma_{j l}^{m}}{\partial \theta_{i}} .
$$

A Jacobi field $X$ along $\gamma^{*}$ is a vector field along $\gamma^{*}$ satisfying the Jacobi equation

$$
\nabla_{\dot{\gamma}^{*}} \nabla_{\dot{\gamma}^{*}} X+R\left(\dot{\gamma}^{*}, X\right) \dot{\gamma}^{*}=0 .
$$

A conjugate point $\gamma^{*}(\tau)$ along $\gamma^{*}$ occurs at time $t=\tau$ if there is a non-trivial Jacobi field $X$ along $\gamma^{*}$ that vanishes at both time 0 and $\tau$, i.e., $X(0)=X(\tau)=0$. We next compute the conjugate points of $\gamma^{*}$ starting from $\gamma^{*}(0)=a$.

Write a vector field $X$ along $\gamma^{*}$ in coordinates as $X=$ $\sum_{i=1}^{k-1} x_{i} \frac{\partial}{\partial \theta_{i}}$ for some $C^{2}$ functions $x_{i}:\left[0, t_{f}\right] \rightarrow \mathbb{R}$, which represents $X$ as a vector $x=\left(x_{1}, \ldots, x_{k-1}\right)$ in $\mathbb{R}^{k-1}$ that varies with time $t \in\left[0, t_{f}\right]$. Then $\nabla_{\dot{\gamma}^{*}} X=$ $\nabla_{\dot{\gamma}^{*}} \sum_{m=1}^{k-1} x_{m} \frac{\partial}{\partial \theta_{m}}=\sum_{m=1}^{k-1} \dot{x}_{m} \frac{\partial}{\partial \theta_{m}}$, and similarly, $\nabla_{\dot{\gamma}^{*}} \nabla_{\dot{\gamma}^{*}} X=\sum_{m=1}^{k-1} \ddot{x}_{m} \frac{\partial}{\partial \theta_{m}}$. On the other hand, since $R$ defined in (13) is a trilinear tensor, by expansion we have

$$
R\left(\dot{\gamma}^{*}, X\right) \dot{\gamma}^{*}=\sum_{i, j, l, m=1}^{k-1} R_{i j l}^{m} x_{j} \frac{\partial}{\partial \theta_{m}} .
$$

So the Jacobi equation (15) along $\gamma^{*}$ is reduced to

$$
\ddot{x}_{m}+\sum_{i, j, l=1}^{k-1} R_{i j l}^{m} x_{j}=0, \quad m=1, \ldots, k-1 .
$$

Equations (16) can be written in matrix form as:

$$
\ddot{x}+B_{k} x=0,
$$

where $B_{k}=\left(b_{m j}\right)_{1 \leq m, j \leq k-1} \in \mathbb{R}^{(k-1) \times(k-1)}$ is a constant matrix with components $b_{m j}=\sum_{i, l=1}^{k-1} R_{i j l}^{m}$.

Calculation show that (see [12] for details)

Lemma $3 B_{k}=\Delta^{-1} \Lambda-I_{k}$, where $\Delta^{-1}$ is given in (9),

$$
\Lambda \triangleq \operatorname{diag}\left(\frac{k-1}{2}, \ldots, \frac{i(k-i)}{2}, \ldots, \frac{k-1}{2}\right)_{1 \leq i \leq k-1},
$$

and $I_{k}$ is the $(k-1)$-by- $(k-1)$ identity matrix.

Solutions of equation (17) are closely related to the spectral decomposition of $B_{k}$, as is summed up in the following two lemmas. Their proofs can be found in [12].

Lemma $4 B_{k}$ has $k-1$ distinctive eigenvalues $\lambda_{j}=$ $\frac{j(j+1)}{2}-1$, for $j=1, \ldots, k-1$. Moreover, eigenvector $v_{j}$ corresponding to $\lambda_{j}$ is of the form

$$
v_{j}=\left[P_{j}\left(\frac{2-k}{k}\right), \ldots, P_{j}\left(\frac{2 i-k}{k}\right), \ldots, P_{j}\left(\frac{k-2}{k}\right)\right]^{T}
$$

for some polynomial $P_{j}$ of degree $j-1$ satisfying

$$
\sum_{x=\frac{2 i-k}{k}, i=1, \ldots, k-1}\left(1-x^{2}\right) P_{l}(x) P_{j}(x)=0, \forall l \neq j .
$$

$P_{j}$ are the discrete version of some orthogonal polynomials called the ultraspherical (or Gegenbauer) polynomials $C_{j-1}^{(\alpha)}$ with parameter $\alpha=\frac{3}{2}$ [16]. In particular, $P_{j}$ is even when $j$ is odd, and odd when $j$ is even.

Lemma 5 The first and last few eigenvectors of $B_{k}$ are

- $v_{1}=[1, \ldots, 1]^{T}$;

- $v_{2}=[2-k, \ldots, 2 i-k, \ldots, k-2]^{T}$;

- $v_{k-2}=\left[(2-k)\left(\begin{array}{l}k \\ 1\end{array}\right), \ldots,(-1)^{i+1}(2 i \quad-\right.$ $\left.k)\left(\begin{array}{c}k \\ i\end{array}\right), \ldots,(-1)^{k}(k-2)\left(\begin{array}{c}k \\ k-1\end{array}\right)\right]^{T}$;

- $v_{k-1}=\left[\left(\begin{array}{c}k \\ 1\end{array}\right), \ldots,(-1)^{i+1}\left(\begin{array}{c}k \\ i\end{array}\right), \ldots,(-1)^{k}\left(\begin{array}{c}k \\ k-1\end{array}\right)\right]^{T}$.

The eigenvectors $v_{1}, \ldots, v_{k-1}$ of $B_{k}$ form a basis of $\mathbb{R}^{k-1}$. Write $x$ in this basis as $x=\sum_{j=1}^{k-1} y_{j} v_{j}$. Then equation (17) becomes

$$
\ddot{y}_{j}+\lambda_{j} y_{j}=0, \quad 1 \leq j \leq k-1 .
$$

Assume that $X$, hence $x$, vanishes at $t=0$. Then $y_{1}(0)=\cdots=y_{k-1}(0)=0$. Non-trivial solutions to the above equations with $y_{j}(0)=0,1 \leq j \leq k-1$, are

$$
\begin{aligned}
& y_{1}(t)=c_{1} t, \\
& y_{j}(t)=c_{j} \sin \left(t \sqrt{\lambda_{j}}\right), \quad j=2, \ldots, k-1,
\end{aligned}
$$

for some constants $c_{1}, \ldots, c_{k-1}$ not identically zero. Conjugate points are encountered at those time epochs $\tau>$ 0 where $y_{j}(\tau)=0$ for all $j$. This is possible only if $c_{1}=0$ and $\tau$ is an integer multiple of $\pi / \sqrt{\lambda_{j}}$ for some $j \in\{2, \ldots, k-1\}$. Therefore,

Theorem 1 The set of conjugate points along $\gamma^{*}$ is

$$
\left\{\gamma^{*}(\tau): \tau=m \pi / \sqrt{\lambda_{j}}, m \in \mathbb{N}, 2 \leq j \leq k-1\right\} .
$$

The first conjugate point along $\gamma^{*}$ occurs at time

$$
\tau_{k} \triangleq \frac{\pi}{\sqrt{\lambda_{k-1}}}=\frac{\pi \sqrt{2}}{\sqrt{(k-2)(k+1)}} .
$$

Note that $\tau_{k} \sim \frac{1}{k}$ as $k \rightarrow \infty$. A geodesic is no longer distance-minimizing beyond its first conjugate point. Thus

Corollary $2 \gamma^{*}$ is not optimal if $t_{f}>\tau_{k}$.

\section{Infinitesimal Perturbations beyond the Conjugate Points}

To find curves shorter than $\gamma^{*}$ with the same end points once $\gamma^{*}$ surpasses its conjugate points, let $\left\{\gamma_{s}^{*}\right\}_{-\epsilon<s<\epsilon}$ be a proper variation of $\gamma^{*}$ in $N$ with variation field $X \triangleq$ $\left.\frac{\partial \gamma_{s}^{*}}{\partial s}\right|_{s=0}$. For each $s$, define $E(s)$ as the energy of $\gamma_{s}^{*}$. By the variation of energy formulas [11], $E^{\prime}(0)=0$ since $\gamma^{*}$ is a geodesic, and

$$
E^{\prime \prime}(0)=-\int_{0}^{t_{f}}\left\langle X, \nabla_{\dot{\gamma}^{*}} \nabla_{\dot{\gamma}^{*}} X+R\left(\dot{\gamma}^{*}, X\right) \dot{\gamma}^{*}\right\rangle d t .
$$




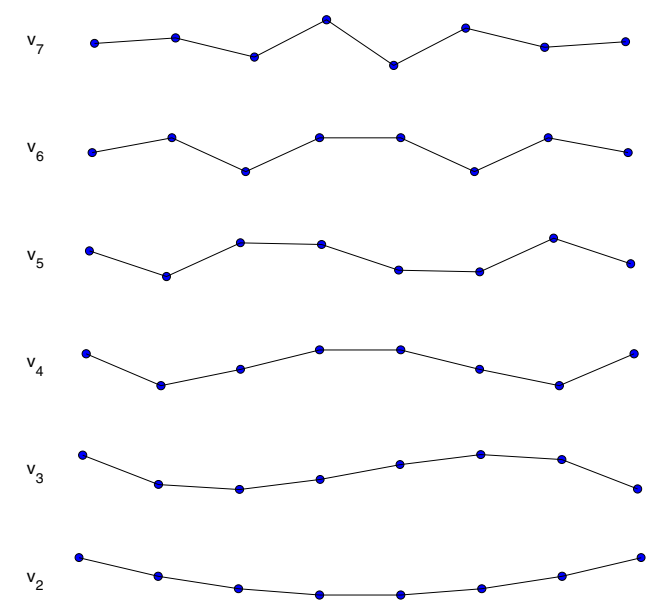

Fig. 4. Perturbed shapes corresponding to $v_{l}, l=2, \ldots, k-1(k=8)$.

Write $X=\sum_{i=1}^{k-1} x_{i} \frac{\partial}{\partial \theta_{i}}$ in the basis $\frac{\partial}{\partial \theta_{1}}, \ldots, \frac{\partial}{\partial \theta_{k-1}}$ along $\gamma^{*}$. Since $\left\{\gamma_{s}^{*}\right\}_{-\epsilon<s<\epsilon}$ is a proper variation, vector $x=\left(x_{1}, \ldots, x_{k-1}\right)$ vanishes at time 0 and $t_{f}$. In this coordinate system, the above equation reduces to

$$
E^{\prime \prime}(0)=-\int_{0}^{t_{f}} x^{T} \Delta\left(\ddot{x}+B_{k} x\right) d t .
$$

Suppose $t_{f}>\pi / \sqrt{\lambda_{j}}$ for some $j \in\{2, \ldots, k-1\}$. Then, by choosing $\left\{\gamma_{s}^{*}\right\}_{-\epsilon<s<\epsilon}$ such that $x(t)=v_{j} \sin \left(\pi t / t_{f}\right)$ where $v_{j}$ is defined in Lemma 4 , we have

$$
E^{\prime \prime}(0)=-\left(\lambda_{j}-\frac{\pi^{2}}{t_{f}^{2}}\right)\left(v_{j}^{T} \Delta v_{j}\right) \int_{0}^{t_{f}} \sin ^{2}\left(\pi t / t_{f}\right) d t
$$

Since $v_{j}^{T} \Delta v_{j}>0$ and $\lambda_{j}-\pi^{2} / t_{f}^{2}>0$, we have $E^{\prime \prime}(0)<0$. Therefore, $\gamma_{s}^{*}$ is shorter than $\gamma^{*}$ for $s$ close to 0 .

To sum up, the above analysis shows that if $t_{f}>\pi / \sqrt{\lambda_{j}}$ for some $j=2, \ldots, k-1$, a solution better than $\gamma^{*}$ can be obtained by infinitesimally perturbing $\gamma^{*}$ so that, $\forall t \in\left[0, t_{f}\right],\left(\theta_{1}, \ldots, \theta_{k-1}\right)$ is incremented by an amount of $v_{j} \sin \left(\pi t / t_{f}\right) d s$. The linked-rod system (snake) formed by connecting successive agents will then assume a shape determined by the signs of the components of $v_{j}$. For example, the alternating signs of the components of $v_{k-1}$ indicate a perturbation where the $k-1$ rods first fold into a saw-like shape during $\left[0, t_{f} / 2\right]$, with the degree of folding of the $l$-th rod from the edge proportional to $\left(\begin{array}{l}k \\ l\end{array}\right)$ for $l=1, \ldots, k-1$, and then straighten up during $\left[t_{f} / 2, t_{f}\right]$. In contrast, $v_{2}$ indicates the $k-1$ rods to bend into a bow-like shape, whereas the shape specified by $v_{k-2}$ is a mixing (product) of the bending specified by $v_{2}$ and the folding specified by $v_{k-1}$. The maximal perturbation occurs at $t=t_{f} / 2$. Fig. 4 plots the various shapes of the linkedrod system at time $t=t_{f} / 2$ caused by the perturbations $v_{j} \sin \left(\pi t / t_{f}\right)$ when $k=8$. Note that these shapes have been rotated to align with the $x$-axis.

The efficiency of the perturbations specified by different $v_{j}$, provided that $t_{f}>\pi / \sqrt{\lambda_{j}}$, can be studied by comparing the respective $E^{\prime \prime}(0)$ under the requirement that $\int_{0}^{t_{f}}\|X\|^{2} d t$ is constant. Since $\int_{0}^{t_{f}}\|X\|^{2} d t=$ $\left(v_{j}^{T} \Delta v_{j}\right) \int_{0}^{t_{f}} \sin ^{2}\left(\pi t / t_{f}\right) d t$, we can conclude by (20) that the larger the eigenvalue $\lambda_{j}$, the more efficient the perturbation specified by its corresponding eigenvector $v_{j}$. Thus the most efficient perturbation is the one given by $v_{k-1}$.

\section{Conclusions}

In this paper, we formulate an optimal formation constrained multi-agent coordination problem and characterize analytically the conjugate points of a geodesic proposed as a candidate solution for a special instance of the problem. We find that this geodesic is optimal for sufficiently close starting and destination positions, but no longer optimal after surpassing its first conjugate point, which occurs from the starting position at a distance that decreases to zero at the same rate as $1 / k$ as the number $k$ of agents increases. In this case, infinitesimally better coordinated motions are determined through a family of orthogonal polynomials.

\section{REFERENCES}

[1] C. Tomlin, G. Pappas, and S. Sastry, "Conflict resolution for air traffic management: a study in multi-agent hybrid systems," IEEE Trans. Automat. Contr., vol. 43, no. 4, pp. 509-521, 1998.

[2] J. P. Desai and V. Kumar, "Nonholonomic motion planning for multiple mobile manipulators," in Proc. IEEE Int. Conf. on Robot. and Automat., vol. 4, Albuquerque, NM, Apr. 1997, pp. 3409-3414.

[3] D. Stipanovic, G. Inalhan, R. Teo, and C. J. Tomlin, "Decentralized overlapping control of a formation of unmanned aerial vehicles," Automatica, vol. 40, no. 8, pp. 1285-1296, 2004.

[4] M. Tillerson, L. Breger, and J. P. How, "Distributed coordination and control of formation flying spacecraft," in Proc. American Contr. Conf., vol. 2, Denver, Co, Jun. 2003, pp. 1740- 1745.

[5] J. D. Wolfe, D. F. Chichka, and J. L. Speyer, "Decentralized controllers for unmanned aerial vehicle formation flight," in Proc. AIAA Guidance, Navigat. and Contr. Conf., San Diego, CA, Jul. 1996.

[6] P. S. Krishnaprasad, "Eulerian many-body problems," in Dynamics and Control of Multibody Systems (Brunswick, ME, 1988). Providence, RI: Amer. Math. Soc., 1989, pp. 187-208.

[7] P. Tabuada, G. J. Pappas, and P. Lima, "Feasible formations of multiagent systems," in Proc. American Contr. Conf., vol. 1, Arlington, VA, Jun. 2001, pp. 56-61.

[8] J. A. Fax, "Optimal and cooperative control of vehicle formations," Ph.D. dissertation, California Institute of Technology, 2002.

[9] N. E. Leonard and E. Fiorelli, "Virtual leaders, artificial potentials and coordinated control of groups," in Proc. 40th IEEE Int. Conf. Decision and Contr., vol. 3, Orlando, FL, 2001, pp. 2968-2973.

[10] R. O. Saber, W. B. Dunbar, and R. M. Murray, "Cooperative control of multi-vehicle systems using cost graphs and optimization," in Proc. American Contr. Conf., vol. 3, Denver, CO, Jun. 2003, pp. 2217- 2222.

[11] M. P. do Carmo, Riemannian Geometry. Boston, MA: Birkhäuser Boston Inc., 1992.

[12] J. Hu, M. Prandini, and C. Tomlin, "Conjugate points in formation constrained optimal multi-agent coordination: A case study," SIAM J. Control and Optimization, submitted for publication. Available upon request.

[13] J. Hu, M. Prandini, and S. Sastry, "Optimal coordinated motions of multiple agents moving on a plane," SIAM J. Control and Optimization, vol. 42, no. 2, pp. 637-668, 2003.

[14] J. W. Milnor, Morse Theory. Princeton, NJ: Princeton University Press, 1963.

[15] J. Hu and S. Sastry, "Optimal collision avoidance and formation switching on Riemannian manifolds," in Proc. 40th IEEE Int. Conf. Decision and Contr, vol. 2, Orlando, FL, Dec. 2001, pp. 1071-1076.

[16] M. Abramowitz and I. A. Stegun, Eds., Handbook of Mathematical Functions with Formulas, Graphs, and Mathematical Tables. New York: Dover Publications Inc., 1992, reprint of the 1972 edition. 